\title{
Response events as well as goal events as sources of animal memory*
}

\author{
E. J. CAPALDI and STEVEN J. HAGGBLOOM \\ Purdue University, W. Lafayette, Indiana 47907
}

\begin{abstract}
Current approaches emphasize the control exercised over behavior by various internal stimuli arising from goal events such as reward and nonreward. In contrast, certain earlier views emphasized the behavioral control exercised by internal stimuli arising from responding. The purpose of the three experiments reported here was to determine if stimuli arising both from goal events and from response events control instrumental behavior, and, if so, how much behavioral control is exercised by each. In the three experiments reported, rats received partial reinforcement in a runway either at a 24 -h ITI or at both a 24 -h ITI and a shorter ITI ( $2 \mathrm{~min}, 15 \mathrm{~min}, 30 \mathrm{~min}$, or $1 \mathrm{~h}$ ), with extinction always occurring at the shorter ITI. The results suggest that both goal-produced and response-produced internal stimuli control behavior simultaneously. Too, it was found that at ITIs as long as 30 min, response-produced cues regulate responding about as strongly as goal-produced cues.
\end{abstract}

Following consistent reward training, the introduction of nonrew arded or smaller rewarded trials will soon disrupt responding. A marked current tendency is to attribute such disrupted responding to the introduction of new goal-related stimuli. That is, generalization decrement or the elicitation of competing responses, or both, results from the introduction of fading traces (e.g., Gonzalez \& Bitterman, 1969), or memories (e.g., Capaldi, 1966), or feedback stimuli of classically conditioned reactions (e.g., Amsel, 1958) occasioned by the new goal events (nonreward or smaller reward). Explanations of this general form may be incomplete, and, moreover, the stimulus control attributed to goal events may actually be exercised in whole, or in part, by other events, such as the animal's responses. That is, the disrupted behavior may, itself, introduce new stimuli which further disrupt behavior. More generally, stimuli produced by the animal's instrumental responses may always regulate instrumental responding.

Two sorts of response-produced cues have been identified-traces of responses made on previous trials (e.g., Hull, 1952) and feedback stimuli of responses currently being executed (e.g., Estes, 1959). Responses may produce memories as well. In any event, many, and perhaps all, operations which have been identified as giving rise to goal-produced stimuli may give rise also (and indeed, in some cases, instead) to response-produced stimuli, although not necessarily merely to stimuli arising from the response classes identified above, a matter covered more fully in the general discussion section. While explanations which have invoked goal-produced cues have almost invariably ignored response-produced cues, and vice versa, Hull (1952) did suggest that successive reversals in discrimination tasks might be controlled by both sorts of

*This research was supported in part by National Institute of Child Health Development Grant 5 R HD04379-03 to E.J. Capaldi. cues, simultaneously. The general view that all instrumental behaviors are always controlled by both sorts of cues, simultaneously, is called here the multiple-control hypothesis."

Animals trained at one intertrial interval (ITI), and then shifted to some different ITI, show behavioral disruption. In keeping with the contemporary emphasis, recent writers (e.g., Amsel, Wong, \& Traupmann, 1971; Capaldi, Berg, \& Sparling, 1971) have at tributed such behavioral disruption to a goal-produced cue, specifically a fading frustrative trace produced by nonreward. An earlier tendency, however, was to attribute such behavioral disruption to a response-produced cue-specifically, a trace produced by the response made on the previous trial (e.g., Rothkopf, 1955; Teichner, 1952). Below, it will be shown that it is at least as plausible to at tribute the behavioral disruption produced by ITI shifts to response-produced cues as to goal-produced cues. The present view, of course, is that both sorts of cues are responsible for the behavioral disruption produced by ITI shifts.

Capaldi et al (1971) reported that resistance to extinction was much greater at a 3-7-min intertrial interval (ITI) in a partial reinforcement (PRF) group trained at that ITI than in a PRF group trained at a 24-h ITI. Capaldi et al (1971) and Amsel et al (1971) attributed this finding to a fading frustrative trace produced by nonreward. According to this view, the frustrative trace which occurred at the 3-7-min extinction ITI was conditioned to the instrumental reaction in the PRF group trained at the 3-7-min ITI, but not in the PRF group trained at the 24-h ITI, the trace having faded completely by $24 \mathrm{~h}$.

Earlier, Teichner (1952) employed consistent reinforcement (CRF) and a shift in ITI from acquisition to extinction, and he reported findings similar to those obtained by Capaldi et al. Under a constant magnitude of CRF, a fading frustrative trace does not occur in acquisition. Thus, the introduction of this trace in 
extinction should produce an equal amount of decrement in CRF groups, regardless of acquisition ITI. Clearly, then, some stimulus other than a frustrative trace was responsible for Teichner's findings. Rothkopf (1955) reported that CRF groups, trained at 24-h ITI, showed behavioral disruption on being shifted to massed-trial CRF. A fading frustrative-trace view fails to explain the findings of Rothkopf and of Teichner.

Rothkopf attributed his findings to a fading trace produced by instrumental responding. That is, while response-produced traces would not be present at a 24 -h ITI, they would be introduced by the shift to the shorter ITI, the longest ITI to which Ss were shifted in the Rothkopf investigation being only $2 \mathrm{~min}$, where they would disrupt behavior. Rothkopf's view, it can be seen, can explain not merely his own results, but also the extinction findings of Teichner following CRF and the extinction findings of Capaldi et al following PRF. Still, it might be thought possible to attribute ITI-shift findings to a goal-produced, rather than to a response-produced, internal stimulus-let us say, to a stimulus trace produced by reward rather than by nonreward. Reward-produced traces can explain Rothkopf's findings because, in that investigation, CRF was employed in the shift phase. However, reward-produced traces cannot be present in extinction and so they cannot explain the extinction findings reported either by Teichner or by Capaldi et al. It does not seem possible, then, to identify a goal-produced cue which is responsible for all of the ITI-shift findings described. The identification of responses as giving rise to the cues in ITI-shift investigations is indirect; responses are common to all of the ITI-shift investigations described, and it would seem that stimuli can arise from the animal's own responses. However, this does not preclude the possibility that some event other than responding is involved, although it is not obvious what this event might be.

No investigation identifying the separate influence of response-produced and goal-produced internal stimuli on some instrumental response has come to our attention. We lack, then, any clear idea of the relative extent to which each of these stimulus classes regulates instrumental behavior. On the one hand, if response-produced cues exercise but slight control over instrumental behavior, they may continue to be safely ignored, at least in most cases. However, if response-produced cues exercise substantial control over instrumental responding, then they will demand increased attention, especially vis-a-vis goal stimuli. A major purpose of the three investigations reported here was to determine the relative extent of behavioral control exercised by goal-produced vs response-produced internal stimuli at various ITIs, ranging from $2 \mathrm{~min}$ to $1 \mathrm{~h}$. A second purpose of the present investigations, mentioned only in passing here, but considered at greater length in the general discussion section, was to determine if the stimuli introduced by an ITI shift, whether goal stimuli or response-produced stimuli, or both, which have generally been considered to be fading traces, might be memories as well. In past investigations in which a reduction of ITI occurred from acquisition to extinction, the extinction ITI was short, on the order of minutes or seconds. In the present investigations, the reduced extinction ITI could be as long as $1 \mathrm{~h}$.

\section{THE PRESENT HYPOTHESIS}

The behavioral control which might be exercised by three internal stimuli was of concern here-that produced by nonreward (called hereafter $S^{N}$ ), that produced by reward ( $\mathrm{SR}^{\mathrm{R}}$ ), and that produced by instrumental responding ( $\mathrm{SI}$ ). For the moment, we may conceptualize $\mathrm{S}^{\mathrm{N}}, \mathrm{SR}^{\mathrm{R}}$, and $\mathrm{S}^{\mathrm{I}}$ as either fading traces or memories. Internal stimuli such as $\mathrm{S}^{\mathrm{N}}, \mathrm{S}^{\mathrm{R}}$, and $\mathbf{S}^{\mathbf{I}}$ occasioned on $\mathrm{Trial} \mathrm{T}$ will be conditioned to the reaction on rewarded $\mathrm{T}+1$ trials but not on nonrewarded $\mathrm{T}+1$ trials (Capaldi, 1967).

Rewarded (R) trials will occasion $S^{\mathbf{R}}$ and $S^{\mathbf{I}}$, while nonrewarded $(\mathrm{N})$ trials will occasion $\mathrm{SN}^{N}$ and $\mathrm{S}^{\mathrm{I}}$. Thus, in transitions from $\mathrm{R}$ trials to $\mathrm{R}$ trials (R-R transitions), $\mathrm{S} R$ and $S^{I}$ acquire control over the reaction, while in transitions from $\mathrm{N}$ trials to $\mathrm{R}$ trials (N-R transitions), $\mathrm{SN}$ and $S^{I}$ will acquire control over the instrumental reaction. Note that neither $S^{R}$ nor $S^{N}$ can be conditioned to the instrumental reaction without simultaneously conditioning $S^{I}$ to the instrumental reaction. In any event, in neither R-N nor $\mathrm{N}-\mathrm{N}$ transitions are stimuli conditioned to the instrumental reaction. However, N-N transitions, in particular, deserve attention, because extinction consists in a series of $\mathrm{N}-\mathrm{N}$ transitions ( $S^{\mathbf{N}}$ and $\mathbf{S}^{\mathbf{I}}$ occur in extinction).

The ITI separating Trial $\mathrm{T}$ and Trial $\mathrm{T}+1$ is said here to determine the value of $S^{N}, S^{R}$, or $S^{I}$. If the ITI is $2 \mathrm{~min}$, the values of the internal stimuli involved will be $2 \mathrm{~min}$. If the ITI is $24 \mathrm{~h}$, we will speak of the $24-\mathrm{h}$ values of whatever internal stimuli are involved. This manner of speaking is not prejudicial; if $S^{N}, S^{R}$, and SI are traces, their 24 -h values will be zero and if they are memories, their 24-h values will be non-zero. If a value of $S^{N}, S^{R}$, or SI is introduced, other than that which has been directly conditioned to the instrumental reaction, response disruption will occur. It seems reasonable to suppose that such disruption will be greater, the greater the disparity between values, and the greater the number of new-valued stimuli introduced.

\section{EXPERIMENT I: MULTIPLE CONTROL}

The purpose of Experiment I was to determine if $\mathbf{S}$, as well as $\mathrm{S}^{\mathrm{N}}$, regulates instrumental behavior, and, if so, the relative extent of behavioral control exercised by each stimulus.

Consider a PRF group trained at a 24-h ITI and then extinguished at some shorter ITI, such as $2 \mathrm{~min}$. This is the sort of PRF group employed by Amsel et al (1971) 
and by Capaldi et al (1971). According to the multiple-control hypothesis, two new-valued stimuli will be introduced in extinction for this group, the $2-\mathrm{min}$ value of $S^{N}$ and the 2-min value of $S^{1}$.

Consider now a PRF group which receives a 24-h ITI between $\mathrm{N}$ trials which are followed by $\mathrm{R}$ trials but only a 2-min ITI between $\mathrm{R}$ trials which are followed by $\mathrm{R}$ trials. For this group, the 2-min value of $S^{I}$ will have been conditioned to the instrumental reaction in acquisition. Thus, only one new-valued stimulus will be introduced for this group at a 2-min extinction ITI, the 2 -min value of $S^{N}$. Accordingly, it may be expected that if $S^{I}$ regulates instrumental responding, then this PRF group will show greater resistance to extinction than a PRF group which received only a 24 -h ITI in acquisition.

\section{Method}

Subjects. The Ss were 48 male albino rats, about 90 days old at the start of the experiment, purchased from the Holtzman Co., Madison, Wisconsin.

Apparatus. The apparatus was a runway, $208.4 \mathrm{~cm}$ long $\mathrm{x}$ $22.9 \mathrm{~cm}$ high $\times 10.2 \mathrm{~cm}$ wide, with a hinged lid of $1.3 \mathrm{~cm}$ hardware eloth and a wooden floor. The alley was divided into three sections. The first section consisted of a treadle, $20.3 \mathrm{~cm}$ long, suspended above a microswitch. By placing the front paws of the rat on the forward edge of the treadle, the first clock $(.01 \mathrm{sec})$ was started. Interruption of a photobeam $5.1 \mathrm{~cm}$ beyond the first photobeam stopped Clock 1 (start time) and activated Clock 2 (run time). Interruption of a photobeam $132.1 \mathrm{~cm}$ beyond the first photobeam stopped Clock 2 and activated Clock 3 (goal time). The goal clock stopped when a photobeam $39.4 \mathrm{~cm}$ beyond the second photobeam and $6.4 \mathrm{~cm}$ in front of a $5.1 \times 10.2 \times 3.8 \mathrm{~cm}$ food tray was broken. Within the food tray was a $3.8 \times 3.2 \times 1.9 \mathrm{~cm}$ metal foodcup which was positioned on the right side of the food tray. A brass guillotine door, $30.5 \mathrm{~cm}$ from the end of the runway, was manually lowered to prevent retracing, once an animal had interrupted the third photobeam.

Design. There were four groups of 12 Ss each. Each group received 20 trials of partial reinforcement. Refer to these as the common trials. Groups $24-0$ and $24-3$ received the common trials at the rate of one trial each day (24-h ITI) and Groups $2-0$ and 2-3 received the common trials at the rate of four each day, the within-day ITI being $2 \mathrm{~min}$. The schedule of rewarded and nonrewarded common trials was as follows: RNNR, NRNR, RNNR, NRNR, and RNNR. Following every fourth common trial, Groups 24-3 and 2-3 received three additional rewarded trials at a 2-min ITI. Groups 24-0 and 2-0 received only the common trials. Note that, in the common trials, rewarded trials never followed rewarded trials at a 2-min ITI.

Experimental Training. Experimental training began for Groups 24-0 and 24-3 on Day 10 and for Groups 2-0 and 2-3 on Day 25. Thus, all four groups entered extinction on the same day. On the days on which they were not run, the Ss in Groups 2-0 and 2-3 were handled briefly. Rats were brought from the colony room to the running room one at a time and, upon completion of the days trials, were returned to the home cage, where 15 min later the daily $12-\mathrm{g}$ ration was provided. Any food eaten in the apparatus was subtracted from the daily ration. Prior to the beginning of all trials, Ss were placed in a neutral waiting box for $2 \mathrm{~min}$. Ss were run in nominal "squad s"-that is, four Ss, one from each group, were run and then four Ss, aga in one from each group, were run, and so on. The running order of the nominal squads was the same each day, but the running order of Ss within a nominal squad was randomly determined each day. On rewarded trials, the goalbox was baited with 10 $.045 \mathrm{~g}$ Noyes pellets and Ss were removed from the goalbox as

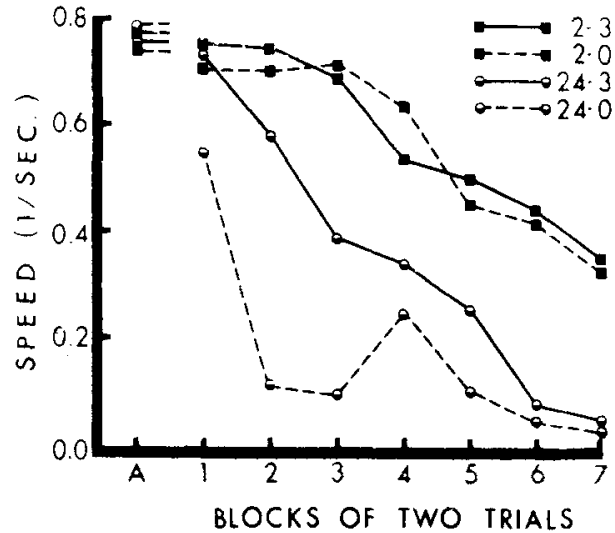

Fig. 1. Running speed along the total length of the alley for each of the four groups employed in Experiment $I$ on the last block of four common trials in acquisition (A) and in blocks of two trials in extinction.

soon as the pellets had been consumed. On nonrewarded trials, $S$ was confined to the unbaited goalbox for $30 \mathrm{sec}$. On all trials, $S$ was placed in the start section of the apparatus and given $60 \mathrm{sec}$ to traverse each alley section. If an $\mathrm{S}$ failed to traverse a particular alley section within $60 \mathrm{sec}$, it was picked up and placed in the goalbox and a 60-sec score was assigned for that alley section, and all forward alley sections, if any. On Day 30 , each $\mathrm{S}$ was given 14 nonreward (extinction) trials at a 2-min ITI. As in acquisition, each $\mathrm{S}$ was run individually.

\section{Results}

In each of the three experiments reported here, similar results both in acquisition and extinction were obtained in each alley section and along the total length of the alley. In each experiment, speeds (1/time) along the length of the alley are presented.

Figure 1 shows speed of running along the total length of the alley for each of the four groups employed in Experiment I on the last block of four common trials in acquisition (A), and in blocks of two trials in extinction. On the last block of common trials in acquisition, differences between the groups were small, although, on prior blocks of common trials, Groups $2-3$ and 24-3 tended to run faster than Groups 2.0 and 24-0, presumably because the former groups received additional rewarded trials. In extinction, groups which received a 2 -min ITI on common trials in acquisition ran faster than groups which received a 24-h ITI on common trials in acquisition, and additional rewarded trials increased resistance to extinction in the 24-h ITI groups but not in the 2-min ITI groups.

The acquisition data shown in Fig. 1 were analyzed, employing a 2 by 2 factorial analysis, the factors being acquisition ITI on the common trials ( 2 min vs $24 \mathrm{~h}$ ) and number of additional rewarded trials ( 0 vs 3 , per block of common trials). On the last block of common trials in acquisition, speed was not influenced, either by acquisition ITI $(F<1)$ or by number of rewarded trials $(\mathrm{F}<1)$. A 2 by 2 factorial analysis was then applied to the mean speeds of each of the four groups over the extinction trials. In extinction, faster running was associated with a 2-min ITI than with a 24-h ITI 
Table 1

Two-Minute Values of $S^{R}, S^{N}$, and $S^{I}$ Conditioned $(X)$ or Not Conditioned (-) in Acquisition

\begin{tabular}{rccc}
\hline & \multicolumn{3}{c}{ Stimuli } \\
\cline { 2 - 4 } Group & $\mathrm{S}^{\mathrm{R}}$ & $\mathrm{SN}$ & $\mathrm{S}^{\mathrm{I}}$ \\
\hline $2-0$ & $\overline{\mathrm{N}}$ & $\mathrm{X}$ & $\mathrm{X}$ \\
$2-3$ & $\bar{X}$ & $\mathrm{X}$ \\
$24-0$ & $\bar{X}$ & - & $\bar{X}$ \\
$24-3$ & - & $\mathrm{X}$ \\
\hline
\end{tabular}

$[F(1,44)=177.98, \mathrm{p}<.001]$, and additional rewarded trials produced faster responding in extinction as well $[F(1,44)=14.24, \mathrm{p}<.001]$. However, reflecting the fact that additional rewarded trials produced faster responding in extinction only at the $24 . \mathrm{h}$ ITI, ITI interacted significantly with additional rewarded trials $[F(1,44)=12.97, p<.001]$. Subsequent Newman-Keuls tests indicated that Group 24-0 differed significantly from all other groups (ps $<.01$ ), as did Group 24-3 (ps $<.01$ ), with no other difference being significant.

\section{Discussion}

In Experiment I, Groups 2-0 and 2-3 were most, and equally, resistant to extinction, and Group 24-3 was more resistant to extinction than Group 24-0. Examined initially are processes and variables, respectively, which could not have been responsible for extinction differences between Groups 24-3 and 24-0. The frustrative trace hypothesis (Amsel et al, 1971; Capaldi et al, 1971) cannot explain this difference, because, in neither Group 24-3 nor Group 24-0 was this trace conditioned to the reaction in acquisition. Too, this difference cannot be explained by pointing to the greater reward percentage, greater number of rewarded trials, and so on, received by Group 24-3, relative to Group 24-0, because all such differences which obtained between these groups also obtained between Groups 2-3 and 2-0, and Groups 2-3 and 2-0 did not differ in extinction.

Groups 24-3 and 24-0 may have differed in extinction because of the introduction of the 2 -min value of $S^{I}$, or because conditioning the 2-min value of $S^{R}$ to the reaction in acquisition provides some generalized basis for responding at a 2-min ITI in extinction, or both. That this difference was due to $S^{I}$, and not to any generalization from $\mathrm{S}^{\mathrm{R}}$, is clear from examining Table 1 , which shows for each group whether the 2-min value of $\mathrm{S}^{\mathrm{R}}, \mathrm{S}^{\mathrm{N}}$, or of $\mathrm{S}^{\mathrm{I}}$ was conditioned to the reaction in acquisition $(\mathrm{X})$ or was not conditioned to the reaction in acquisition (-).

Of great importance is understanding why the 2-min value of $\mathrm{S}^{\mathrm{R}}$ was not conditioned to the reaction Group 2-0. In Group 2-0, rewarded trials at a 2-min ITI were always followed by nonrewarded trials, and neither $\mathbf{S}^{\mathbf{R}}$ nor any stimulus is conditioned to the reaction on nonrewarded trials (e.g., Capaldi, 1966). Now, if the extinction difference between groups $24-3$ and $24-0$ was due to the 2-min value of $S^{R}$, then Groups 2-3 and 2-0 would have differed in extinction, because these groups also differed along the $S^{R}$ value dimension. But, if the extinction difference between Groups 24-3 and 24-0 was due to the 2 -min value on SI, then, as found here, Groups 2-3 and 2-0 should not have differed in extinction, because in both groups the 2-min value of $\mathbf{S}^{1}$ had been conditioned to the instrumental reaction. It is clear from Table 1, then, that the difference in extinction between Groups 24-3 and 24-0 must have been due either exclusively or primarily to $S^{\mathrm{I}}$, and only secondarily, if at all, to generalization from $S^{R}$. A better estimate of the behavioral control exercised by $S^{R}$ in extinction would be available if a group having the 24-h values of $S^{N}$ and of $S^{R}$, but the 2-min value of $S^{I}$ conditioned to the reaction, had been included in Experiment I. However, we know of no way that this can be accomplished.

An estimate of the extent to which $S^{N}$ and $S^{I}$ controlled behavior in extinction is available from Table 1. The difference in extinction between Groups 2-3 and 24-3 was due, as can be seen in Table 1, to $\mathrm{S}^{\mathrm{N}}$. As indicated, the difference in extinction between Groups $24-3$ and 24-0 was due to SI. As Fig. 1 shows, the difference in extinction between Groups 2-3 and 24-3 (due to $\mathrm{S}^{\mathrm{N}}$ ) was about as large as the difference in extinction between Groups 24-3 and 24-0 (due to $S^{I}$ ). It appears, then, that in extinction, $\mathrm{S}^{\mathrm{I}}$ controlled behavior to a considerable extent, about as strongly as $\mathrm{S}^{\mathrm{N}}$. That $\mathrm{S}^{I}$ so strongly controlled responding in extinction may be regarded as surprising, in view of the current emphasis on goal-related stimuli such as $\mathrm{S}^{\mathrm{N}}$. It would seem clear, on the basis of the present results, that the reduced resistance to extinction shown by PRF groups trained at a 24-h ITI and then extinguished at a short ITI in the investigations of Amsel et al (1971) and Capaldi et al (1971), and attributed by them to a fading trace produced by nonreward, was due as much to $\mathrm{SI}^{\mathrm{I}}$ as to any stimuli which were produced by nonreward. The general question arises, in how many other cases are results which have been attributed to goal-produced stimuli such as $\mathrm{S}^{\mathbf{R}}$ and $\mathrm{S}^{\mathrm{N}}$, due as well to SI? In the general discussion section, it will be suggested that all reward reduction phenomena, such as the partial reinforcement extinction effect, the successive negative contrast effect, and so on, may be mediated by response-produced stimuli, as well as by goal-produced stimuli. The basis of this suggestion is that response-produced cues may be conditioned to the reaction under PRF which are both different from and more capable of eliciting responding in extinction than the response-produced cues conditioned to the reaction under CRF. It will be recognized that this reasoning is of the general form which has been applied to goal-produced cues, i.e., it is generally accepted (see Amsel, 1958; Capaldi, 1966) that goal-produced cues are 
Table 2

Rewarded (R) and Nonrewarded (N) Trials for Groups NR, RR, and Con-15

\begin{tabular}{ll}
\hline Group & Schedule \\
\hline NR & R NR R NR R NR R R R NR R R NR R NR R NR R R NR R R R NR \\
R NR R NR R R NR R \\
RR \\
R N RR N RR N RR R R N RR R N RR N RR N RR R N RR R R N \\
RR N RR N RR R N RR \\
Con-15 & RNRR NRRNRR RRNRR RNRR NRRNRR RNRR RRNRR NRRNRR RNRR \\
\hline
\end{tabular}

Note-A space between trials indicates a 24-h ITI; no space indicates a 15-min ITI.

conditioned to the reaction under PRF which are both different from, and more capable of, eliciting responding in extinction than the goal-produced cues conditioned to the reaction under CRF.

\section{EXPERIMENT II}

In terms of underlying logic, Experiment II is identical to Experiment $I$. That is, in terms of extinction values of $\mathbf{S}^{\mathbf{R}}, \mathrm{S}^{\mathbf{I}}$, and $\mathbf{S}^{\mathbf{N}}$, conditioned or not conditioned to the instrumental reaction in acquisition, each of the four groups employed in Experiment II corresponds to one of the groups employed in Experiment I. However, logic aside, there were two noteworthy differences between Experiments I and II. First, the relative extent of behavioral control exercised by $S^{I}$ and $S^{N}$ at a 15 -min ITI was determined in Experiment II rather than, as in Experiment I, at a 2-min ITI.

More importantly, perhaps, in Experiment II the specific conditions under which internal stimuli did or did not acquire control over the instrumental reaction differed, and sometimes very substantially, from the experimental conditions employed in Experiment I. This was done in order to determine if the results obtained in Experiment I were peculiar somehow to the experimental conditions employed in Experiment I. If they were not, that is, if roughly the same results were obtained in Experiment II as were obtained in Experiment I, it would become likely that the multiple-control hypothesis could be confirmed under a wide variety of experimental conditions. The effect of this, of course, would be to exclude, at least to a degree, interpretations other than the multiple-control hypothesis which might be compatible with the results obtained in Experiment I. For example, while the results obtained in Experiment I suggest otherwise, reward percentage or number of rewarded trials might be thought by some to have had some effect in Experiment I, which recommends against accepting the multiple-control hypothesis. In Experiment II, all groups received the same reward percentage, the same number of rewarded trials, and so on. As another example, contiguity theorists might be disinclined to accept the conclusion offered earlier that in Group 2-0 the 2-min value of $\mathrm{SR}^{\mathrm{R}}$ was not conditioned to the instrumental reaction in acquisition because, in Group 2-0, rewarded trials were followed by nonrewarded trials at a 2-min ITI. Group NR of Experiment II is similar to Group 2-0 of Experiment $I$ in that the extinction values of $S^{N}$ and of $S^{I}$, but not of $S^{R}$, were conditioned to the reaction in acquisition (see Table 1). However, the procedure employed in preventing the extinction value of $S^{R}$ from acquiring control over the reaction was different in the two cases. Specifically, in the case of Group NR, rewarded trials were not followed by any trial, either rewarded or nonrewarded, at the extinction ITI. Thus, neither from a contiguity nor from a reinforcement viewpoint was the extinction value of $\mathrm{S}^{\mathrm{R}}$ conditioned to the instrumental reaction in Group NR.

\section{Method}

Subjects. The Ss were 48 male albino rats purchased from the Holtzman Co., Madison, Wisconsin, about 90 days old at the start of the experiment.

Apparatus. The same apparatus was employed in Experiment II as was used in Experiment I.

Design. There were four groups of 12 Ss each. Each group received 44 trials of partial reinforcement, the schedule of rewarded $(\mathrm{R})$ and nonrewarded $(\mathrm{N})$ trials being the same in each group. The groups differed in terms of the ITI separating various trials. One group received a $24-\mathrm{h}$ ITI between all trials. This is a conventional 24-h ITI group (Group Con-24). The ITIs separating various trials in the remaining groups are shown in Table 2.

In Table 2, a space between trials, e.g., $R \mathrm{~N}$, indicates a $24-\mathrm{h}$ ITI between trials, and a lack of a space between trials, e.g., NR indicates a 15-min ITI. As can be seen in Table 2, Group NR received a 15 -min ITI between nonrewarded trials, followed by rewarded trials, and a 24-h ITI between all rewarded trials, followed by rewarded trials. Group RR received the same number of trials, separated by a 15-min ITI as Group NR; however, in Group RR, it was rewarded trials followed by rewarded trials which were separated by a 15-min ITI. Group Con-15 was a conventional 15-min partial-reward group. Group Con-15 received either 4, 5, or 6 daily trials; this was done so that Group Con-15 would receive exactly the same number of transitions from nonrewarded to rewarded trials at a 15 -min ITI as Group NR. Note too that Group Con- 15 received about as many transitions from rewarded trials to rewarded trials as did Group RR (14 vs 12).

Preliminary Training. On Day 1 , the rats were assigned to individual cages and were fed $12 \mathrm{~g}$ of Wayne Lab-blox, the deprivation schedule employed throughout the experiment. The rats were handled on Days 8-12. Because the groups did not receive the same number of trials each day and because it was considered mandatory that all groups enter extinction on the same day, experimental training began on different days for the groups, Day 18 for Group Con-24, Day 30 for Groups NR and 


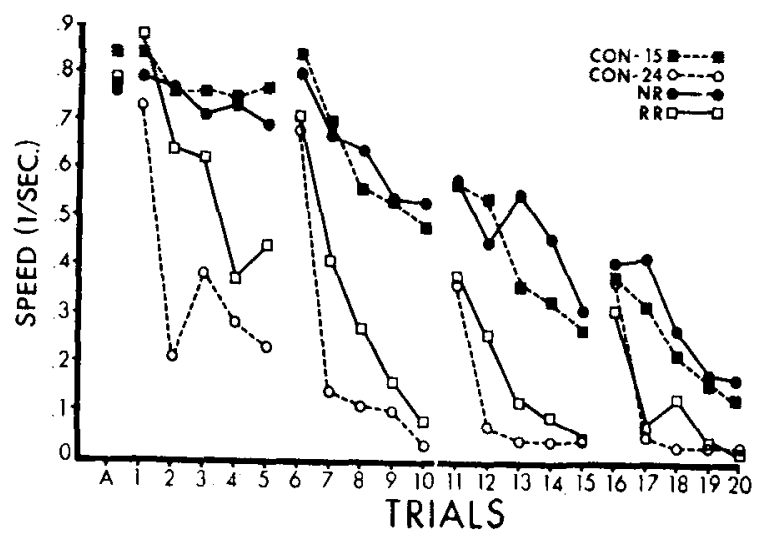

Fig. 2. Running speed along the total length of the alley on the last four trials of acquisition (A) and on each of the trials of each day of extinction for each of the four groups employed in Experiment II.

RR, and Day 53 for Group Con-15. On the 5 days immediately prior to the start of experimental training, each $S$ received one daily rewarded runway trial of 15 pellets, the reward magnitude employed throughout the experiment. On all rewarded trials, $S$ was removed from the goalbox as soon as the food was eaten and food eaten in the apparatus was subtracted from the daily ration.

Experimental Training. When only Ss from Group Con-24 were run (Days 18-29), Ss were assigned to nominal squads of three each. The order of running Ss within a nominal squad was randomized daily, and the order of running nominal squads within a day was constant. With the introduction of the NR and RR Ss on Day 30, two different procedures for running the Ss were employed, depending upon whether all Ss received one trial on that day or one of the groups (NR or RR) received two trials on that day. On one-trial days, the Ss were run in nominal squads of three, one $S$ from each group, the running order of $S$ s within a nominal squad being randomly determined, with the order of running nominal squads within a day being constant. On two-trial days, the nominal squads were composed of one $S$ from Group Con-24, two Ss from the group receiving two trials on that day (NR or RR), and one $S$ from either Group RR or Group NR, whichever was to receive one trial on that day. The two Ss from this nominal squad which were to receive two trials on that day did not receive their second trial until Ss from two other nominal squads of the type described received their first trial. Thereupon, these Ss received their second trial in a new nominal squad of four Ss, composed of the two Ss mentioned, one $S$ from Group Con-24 and one $S$ from the remaining group. This procedure was repeated. It allowed $15 \mathrm{~min}$ to elapse between trials for the two trial Ss of that day and it eliminated systematic factors such as always running Ss from a particular group following Ss of another group and so on. With the introduction of Group Con-15 on Day 53, the following procedure was observed on days that Group Con-15 was to receive four trials. An $S$ from Groups $R R$ or NR which was to receive two trials was run. An $S$ from Group Con-15 was then run. Before this $S$ received its second trial, 11 other $S s$ were run, 5 from Group Con- 15 min, 4 from the group to receive two trials that day (NR or RR), and $1 \mathrm{~S}$ from each remaining group on half the occasions. On the day neither Group RR nor Group NR was to receive two trials, or on days when Group Con-15 was to receive more than four trials, appropriate variants of this proced ure were employed.

On Days 62-65, each $S$ received five extinction trials at a 15-min ITI. The Ss were run in squads of $12 \mathrm{each}$, three Ss from each group comprising a squad. Every $S$ within a squad received its first trial before the next $S$ received its second trial, and so on. The running order of Ss within a squad was randomized daily. On all nonrewarded trials, both in acquisition and extinction, $S$ was confined to the empty goalbox for $20 \mathrm{sec}$. On all trials, $S$ was placed in the start section of the apparatus and given $30 \mathrm{sec}$ to traverse each alley section. If an $\mathrm{S}$ failed to traverse a particular alley section within $30 \mathrm{sec}$, it was picked up and placed in the goalbox and a 30-sec score was assigned for that alley section and all forward section, if any. In Experiment II, unlike as in Experiment I, the Ss were housed in the running room. Ss were housed in the home cage between trials and were fed the daily ration minus any food eaten in the apparatus $15 \mathrm{~min}$ following the last daily trial.

\section{Results}

Figure 2 shows speed of running along the total length of the alley on the last four trials of acquisition (A) and on each of the trials of each day of extinction for each of the four groups employed in Experiment II. Differences between the groups were small at the end of acquisition. In extinction, Groups NR and Con-15 differed from each other only minimally, and these groups ran faster than Group RR, which, in turn, ran faster than Group Con-24. The speeds of Group Con-24 dropped substantially on the second extinction trial of each day. Prior to extinction, Group Con-24 had not experienced a trial at a $15 \cdot \mathrm{min}$ ITI.

A simple between-groups analysis of variance was applied to the data shown in Fig. 2. Differences between the groups at the end of acquisition were highly significant $[F(3,44)=30.26, p<.001]$. Subsequent Newman-Keuls tests indicated that while Groups NR and Con-15 did not differ in extinction, every other pair of groups differed at the .05 level or better.

\section{Discussion}

It was found, in Experiment II, that Groups NR and Con-15 were equally resistant to extinction and more resistant to extinction than Group $R R$, which was, in turn, more resistant to extinction than Group Con-24. Table 3 shows, for each group employed in Experiment II, whether the $15-\mathrm{min}$ values of $\mathrm{S}^{\mathrm{R}}, \mathrm{S}^{\mathrm{N}}$, or SI were conditioned to the instrumental reaction in acquisition $(X)$ or were not conditioned to the instrumental reaction in acquisition $(-)$.

An inspection of Table 3 and of Table 1 will show that, in terms of the extinction values of $S^{R}, S^{N}$, and $S^{I}$

Table 3

Fifteen-Minute Values of $\mathbf{S}^{\mathbf{R}}, \mathbf{S}^{\mathbf{N}}$, and $\mathbf{S}^{\mathbf{I}}$ Conditioned $(X)$ or Not Conditioned (-) in Acquisition

\begin{tabular}{lccc}
\hline & \multicolumn{3}{c}{ Stimuli } \\
\cline { 2 - 4 } Group & $\mathrm{S}^{\mathrm{R}}$ & $\mathrm{S}$ & $\mathrm{S}^{\mathbf{I}}$ \\
\hline NR & - & $\mathrm{X}$ & $\mathrm{X}$ \\
Con-15 & $\mathrm{X}$ & $\mathrm{X}$ & $\mathrm{X}$ \\
Con-24 & - & - & - \\
RR & $\mathrm{X}$ & - & $\mathrm{X}$ \\
\hline
\end{tabular}


conditioned or not conditioned to the instrumental reaction in acquisition, Groups NR, Con-15, Con-24, and RR of Experiment II correspond, respectively, to Groups 2-0, 2-3, 24-0, and 24-3 of Experiment I. Thus, the results obtained in Experiment II can be seen to be remarkably similar to those obtained in Experiment I. Accordingly, the results obtained in Experiment II suggest that at a 15 -min ITI, $\mathrm{SI}$, as well as $\mathrm{S}^{\mathrm{N}}$, controls behavior, and that the behavioral control exercised by each at a $15 \cdot \mathrm{min}$ ITI is approximately equal. The groups employed in Experiment I, but not in Experiment II, differed along such dimensions as number of rewarded trials, number of trials, percentage of reward, and so on. Thus, these variables have been shown as unimportant to the results obtained in Experiment I, not merely by the control procedures employed in Experiment I, but as well by the results obtained in Experiment II.

Of considerable interest in Experiment II is the extinction behavior of Group Con-24. As can be seen in Fig. 2, while Group Con-24 ran relatively rapidly on Trial 1 of each extinction day (24-h values of $S^{\mathrm{I}}$ and of $\mathrm{SN}^{\mathrm{N}}$ ), on Trial 2 , and trials subsequent, of each extinction day, Group Con-24 showed a substantial speed drop. That is, from the outset of extinction, Group Con-24 showed little tendency to respond in the presence of the 15 -min values of $\mathrm{SI}^{\mathrm{I}}$ and of $\mathrm{SN}^{\mathrm{N}}$. The behavior of Group Con-24 clearly indicates that the capacity of the 15 -min values of $\mathrm{S}^{\mathrm{I}}$ and of $\mathrm{SN}^{\mathrm{N}}$ to elicit the reaction is practically nil, if only the 24 -h values of these stimuli have been conditioned to the instrumental reaction. Accordingly, the difference between the 15-min and 24 -h values of these stimuli must be substantial,

\section{EXPERIMENT II}

Experiment II suggested that $S^{I}$ and $S^{N}$ have $15-m i n$ values which differ substantially from the 24 -h values of these stimuli, and that at a 15 -min ITI, response-produced stimuli control behavior about as strongly as goal-produced stimuli. Experiment III was concerned with the same matters as Experiment II except at a 30-min and at a 1-h ITI. The groups employed in Experiment III received the same training as Groups NR, RR, and Con-24 of Experiment II except either at a 30-min ITI (Groups NR-30, RR-30, and Con-24-30) or at a 1-h ITI (Groups NR-60, RR-60, and Con-24-60). Unlike Experiment II, which included a conventional 15-min PRF group, Experiment III did not include conventional 30-min or 60-min PRF groups.

\section{Method}

Subjects. The Ss were 60 male albino rats purchased from the Holtzman Co., Madison, Wisconsin. They were about 90 days old at the start of the experiment.

Apparatus. The same apparatus was employed in Experiment III as was used in Experiments I and II.

Design. There were six groups of $10 \mathrm{Ss}$ each. These groups received the same schedule of partial reward as was employed in
Experiment II and which is shown in Table 2. There were two NR groups, two RR groups, and two Con-24 groups, and these were treated exactly as were the corresponding groups of Experiment II, with one exception: the ITI was not $15 \mathrm{~min}$, as in Experiment II, but was either 30 min (Groups NR-30, RR-30, and Con-24-30) or $60 \mathrm{~min}$ (Groups NR-60, RR-60, and Con-24-60).

Preliminary Training. The rats were assigned to individual cages on Day 1 and were fed $12 \mathrm{~g}$ of Wayne Lab-blox daily. Acquisition training began on Day 18 for Groups Con-24-30 and Con-24-60 and on Day 30 for the remaining groups so that all groups might enter extinction on Day 62. Pretraining began 10 days prior to acquisition training. It consisted of 5 days of handling, followed by 2 days of feeding Ss $.045-\mathrm{g}$ Noyes pellets in the home cage, followed by one daily rewarded trial (15 pellets) for 3 days. Ss were always removed from the goalbox after the food had been eaten, and the amount of food fed in pellets was always subtracted from the daily ration. Reward in the goalbox was always 15 pellets.

Experimental Training. On Days 18-29, only Groups Con-24-30 and Con-24-60 were run. On these days, the Ss were run in a randomized order each day. With the introduction of the remaining four groups on Day 30 , two procedures for running the Ss were employed, depending upon whether all groups received 1 trial on a particular day or two of them received 2 trials. On 1-trial days, the $S s$ were run in nominal squads of six, one from each group, the running order of Ss within a squad being randomly determined with the order of running nominal squads over the day being fixed. On 2-trial days, the procedure was as follows: 9 were receive 2 trials was run first, and that $S$ received its second trial after 48 other trials had been run. The 48 trials were as follows; 9 were those of the remaining Ss in the $60-\mathrm{min}$ group to receive 2 trials, 10 were the first and second trials of $30-\mathrm{min}$ Ss to receive 2 trials that day, 5 were the first trials of the remaining five Ss from the 30-min group to receive 2 trials that day, and 24 were from six Ss in each of the remaining four groups which were to receive 2 trials that day. There were then 30 more trials to be run; 9 were the second trials of the $60-\mathrm{min}$ group receiving 2 trials that day, 5 were the second trials of the 30 -min group receiving 2 trials that day, and 16 were those of four Ss, one from each of the remaining four groups.

All Ss received four extinction trials each day for 5 days. Confinement on nonrewarded trials was $15 \mathrm{sec}$. The 60 -min ITI groups were run in a single squad of $30 \mathrm{Ss}$, with running order being randomized daily within 10 subsquads of $10 \mathrm{Ss}$ each. Interspersed among the 60-min Ss were the 30-min Ss, which were run in two squads of $15 \mathrm{Ss}$, running order being randomized daily within five subsquads of three Ss each. In the event an $S$ failed to respond in a particular alley section within $30 \mathrm{sec}$, it was placed in the goalbox and a time score of $30 \mathrm{sec}$ was assigned for that alley section and for all forward alley sections. The Ss were housed in the home cage between trials and were fed the daily $12-\mathrm{g}$ ration minus any amount eaten in the apparatus $15 \mathrm{~min}$ following the last daily trial.

\section{Results}

Figure 3 shows speed of running along the total length of the alley on the last four trials of acquisition (A) and on each of the trials on each day of extinction for the groups extinguished at a 30 -min ITI (left panel) and for the groups extinguished at a 60-min ITI (right panel). Differences between the groups at the end of acquisition were minimal. The ordering of groups in extinction obtained in Experiment II, NR followed by RR, followed by Con-24, was obtained in Experiment III at the 30 -min ITI, but at the $60-\mathrm{min}$ ITI, while the NR 


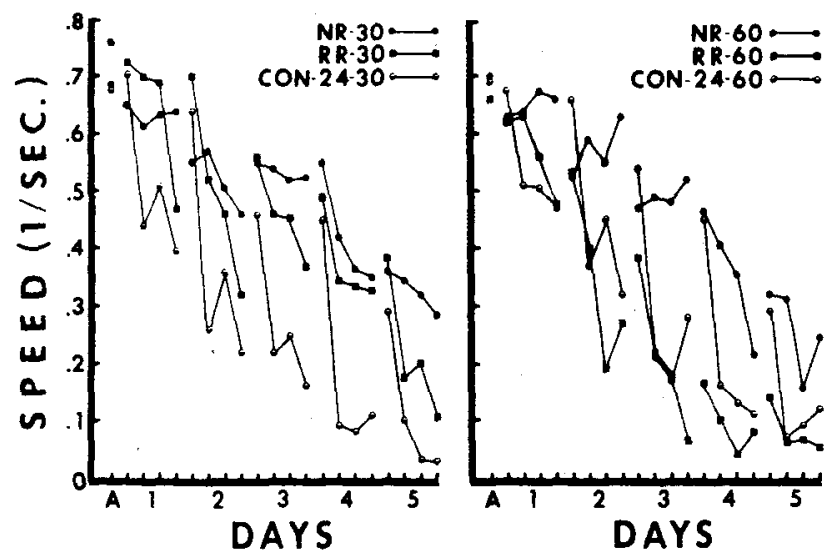

Fig. 3. Running speed along the total length of the alley on the last four trials of acquisition (A) and on each of the trials of each day of extinction for the groups extinguished at a $30-\mathrm{min}$ ITI (left panel) and for the groups extinguished at a 60 -min ITI (right panel).

group was most resistant to extinction, the $R R$ and Con-24 groups differed only minimally, and, indeed, overall, at the 60-min ITI, the RR group was, if anything, slower than the Con-24 group.

A 2 by 3 factorial analysis of variance was applied to the data shown in Fig. 3, the factors being extinction ITI (30 vs $60 \mathrm{~min}$ ) and acquisition treatment (NR vs RR vs Con-24). Responding in acquisition was not affected by ITI $(\mathrm{F}<1)$, by acquisition treatment $(\mathrm{F}<1)$, or by the interaction of these variables $(F<1)$. In extinction, differences due to ITI were not significant $[F(1,54)=2.08, p>.10]$. Differences due to the acquisition treatment were significant $[\mathrm{F}(2,43)=7.98$, $\mathrm{p}<.002]$. The Acquisition Treatment by ITI interaction was not significant $[F(2,43)=2.98$, $.05<\mathrm{p}<.10$ ). Planned comparisons indicated that, in extinction, Group NR-30 ran faster than either Group RR-30 $[\mathrm{F}(1,54)=4.02, \mathrm{p}<.05)$ or Group Con-24-30 $[\mathrm{F}(1,54)=10.01, \mathrm{p}<.01]$, and that Group RR-30 ran faster than Group Con-24-30 $[F(1,54)=5.27, p<.05]$. However, at the 60-min ITI, while Group NR-60 ran faster than either Group RR-60 $[F(1,54)=10.80, p<.01]$ or Group Con-24-60 $[F(1,54)=8.31, p<.01]$, Groups RR-60 and Con-24-60 did not differ in extinction $[F(1,54)=2.82$, $\mathrm{p}>.10]$.

\section{Discussion}

In Experiment III, Group Con-24-30 extinguished more rapidly than Group RR-30, and Group RR-30 extinguished more rapidly than Group NR-30. Further, the difference between Groups Con-24-30 and RR-30 was about as large as that between Groups Con-24-30 and NR-30. These results suggest that $S^{I}$ and $S^{N}$ have $30-\mathrm{min}$ values, that the $30-\mathrm{min}$ values of $\mathrm{S}^{\mathrm{I}}$ and $\mathrm{S}^{\mathrm{N}}$ differ from the 24-h values of these stimuli, and that at a 30-min ITI, about the same extent of behavioral control is exercised by $\mathrm{SI}^{\mathrm{I}}$ as by $\mathrm{S}^{\mathbf{N}}$. In Experiment III, Group NR-60 was more resistant to extinction than Groups Con-24-60 or RR-60, which did not differ in extinction. These results suggest that $S^{N}$ has a $1-h$ value which differs from the 24-h value of $S^{N}$.

The failure of Groups RR-60 and Con-24-60 to differ in extinction suggests that the 1-h and $24-\mathrm{h}$ values of $\mathrm{SI}^{\mathrm{I}}$ do not differ, or at least they differ only minimally. From this lack of difference, little can be inferred concerning whether, at a $1-\mathrm{h}$ ITI, SI has or does not have some nonzero value. That is, regardless of whether the 1-h and 24-h values of SI are zero or nonzero, so long as the two values are the same, Groups RR-60 and Con-24-60 would not be expected to differ in extinction. Of course, it makes considerable difference whether the 1 -h value of $S^{I}$ is or is not zero. Among other things, if the $1-h$ value of $S^{I}$ were zero, then $S^{I}$ would not regulate behavior at ITIs of about $1 \mathrm{~h}$ and longer. In any case, it would appear that at ITIs of about $1 \mathrm{~h}, \mathrm{~S}^{\mathrm{N}}$ may exercise greater control over instrumental responding than SI. This would certainly be the case if the 1-h value of $\mathrm{S}^{\mathrm{I}}$ is zero. If the 1 -h value of $\mathrm{S}^{\mathrm{I}}$ is not zero, and if the 1-h and 24-h values of SI do not differ, then it cannot be determined from Experiment III how much behavioral control was exercised by $\mathrm{S}^{\mathrm{I}}$ at a 1 -h ITI.

One need not be a trace theorist in order to assume that there is an ITI which is shorter than $24 \mathrm{~h}$, which will provide a value of $S^{\mathbf{N}} ; S^{\mathbf{R}}$, or of $\mathrm{S}^{\mathbf{I}}$, which is not much different from the 24-h values of these stimuli. Too, in order to obtain a difference between, for example, Group NR-60 and Group RR-60, the 1-h and 24-h values of $S^{N}$ must not merely differ, but they must be discriminably different for the rat. Given these observations, the results obtained in the present investigation suggest that the rat's capacity to discriminate between various values of $S^{N}$ and $S^{I}$ is rather well developed. However, it appears that $\mathrm{S}^{\mathrm{I}}$ grows nondiscriminable from its 24 h value within a shorter span of time ( $30 \mathrm{~min}$ to $1 \mathrm{~h}$ ) than does $\mathrm{S}^{\mathrm{N}}$ (beyond $1 \mathrm{~h}$ ).

\section{GENERAL DISCUSSION}

As indicated earlier, a current tendency is to explain a variety of instrumental behaviors in terms of the behavioral control exercised by goal-produced stimuli. The present results suggest that the behavioral decrement which occurs on a shift from a longer ITI in acquisition to a shorter ITI in extinction, which can be ascribed either to goal-produced stimuli (Amsel et al, 1971; Capaldi et al, 1971) or to response-produced stimuli (Rothkopf, 1955; Teichner, 1952), is probably due to both sources of stimulus control, at least at IT Is of up to about $30 \mathrm{~min}$. Given these findings, a question arises as to what extent other phenomena attributed to goal-produced stimuli in the contemporary literature might be due, as well, to response-produced stimuli. At 
the present, too little is known about response-produced cues to supply a specific answer to this question. What can be done, however, is to describe the general conditions under which behavioral control that has been ascribed to goal-produced stimuli might also have been exercised by response-produced stimuli. It would be well also to show, by means of examples, that these general conditions have been met at least in some concrete experimental settings. Certain reward-schedule effects are selected for this purpose here.

Response-produced cues could not influence instrumental responding if distinctive responses, such as right vs left turning (see Hull, 1952) or fast vs slow running, and so on, did not give rise to distinctive stimuli. Later on, we shall deal with what sorts of response events might give rise to distinctive cues. The cues occasioned by responses may be those arising from responses made on the previous trial or trials (traces or memories) or those arising from responses currently being made. Label the former class of response-produced cues $S^{I}$ and the latter class $S_{I}$. If findings attributed to goal-produced cues are due in some part to response-produced cues, then it necessarily must be that the manipulation of goal events gives rise to variations of one sort or another in response events. Let us satisfy ourselves that this second requirement is met when schedule of reward is manipulated.

In early acquisition trials, PRF animals run slowly following nonrewarded trials, more slowly than following rewarded PRF trials, and more slowly than do CRF animals following comparable rewarded trials (e.g., Capaldi \& Hart, 1962; Capaldi, Turner, \& Wynn, 1962; Tyler, Wortz, \& Bitterman, 1953). Thus, conditioned to the reaction under PRF, but not under CRF, may be not merely $\mathrm{SN}^{\mathrm{N}}$, but stimuli characteristic of slow running as well. Too, in early acquisition, PRF animals run progressively more slowly as the number of consecutive nonrewarded trials increases (Capaldi \& Hart, 1962; Capaldi et al, 1962). This variable has been called $\mathrm{N}$-length, and resistance to extinction increases as $\mathrm{N}$-length increases (see Capaldi, 1966). There is a greater tendency, then, under a PRF schedule than under a CRF schedule to have both $S_{I}$ and $S^{I}$ characteristic of slow running associated with reward. Let us be even more specific. A PRF animal which receives, for example, the sequence of nonrewarded $(\mathrm{N})$ and rewarded $(\mathrm{R})$ trials, NRR, will run relatively slowly in early acquisition on the first $R$ trial and will run relatively rapidly on the second $R$ trial. On the first $R$ trial, $S_{I}$ characteristic of slow running will be associated with reward, and on the second R trial, $\mathrm{S}^{\mathrm{I}}$ characteristic of slow running will be associated with reward. The second $R$ trial is of particular interest, for on that trial, $S^{\mathbf{I}}$ characteristic of relatively slow running will occur in combination with relatively rapid running. Thus, $S^{I}$ characteristic of relatively slow running can, under PRF, become a cue for the elicitation of relatively rapid running.
It becomes possible, then, that when the PRF animal runs slowly in extinction, the stimuli occasioned by such slow running may, on the subsequent trial, evoke fast running. For the CRF animal, however, cues produced by slow running will not elicit the reaction because they were not conditioned to the instrumental reaction in acquisition, and, indeed, cues produced by slow running in the CRF animal may produce generalization decrement. Thus, once the CRF animal begins to run slowly in extinction, the cues produced by such slow running may elicit still slower running, which, in turn, may elicit still slower running, and so on, generating in extinction for the CRF animal what may be called a snowball effect. This snowball effect, as we have seen, would be attenuated for the PRF animal. Of course, not merely in extinction, but in any case involving reward reduction, such as the negative contrast effect, response-produced cues may be a factor regulating responding.

The manipulation of goal events may give rise to still other distinctive response-produced cues, which may be even more responsible for differences between, for example, PRF and CRF groups, than those considered above. Rewarded and nonrewarded trials differ, not merely in terms of the presence or absence of reward, but also in terms of the distinctive goalbox behaviors which occur on those trials. If these distinctive behaviors give rise to distinctive $\mathrm{S}^{\mathrm{I}}$ cues, it is clear that such behaviors would contribute to greater resistance to extinction following PRF than following CRF. That is, there is opportunity under PRF, but not under CRF, for distinctive cues associated with nonrewarded goalbox behaviors to acquire control over the reaction. Accordingly, when these cues are introduced in extinction, they elicit the reaction in PRF animals, but not in CRF animals. The fact that distinctive goalbox behaviors as well as different runway behaviors, or more generally distinctive behaviors pripr to the receipt of the goal object, might give rise to distinctive $S^{I}$ cues has been overlooked.

Estes (1959) heavily emphasized response-produced cues in his attempt to deal with reward schedule effects. There are, however, many points of difference between the response-produced cue analysis described above, which, as will be suggested below, is based upon memory, and the response-produced cue analysis suggested by Estes, which is based on classical conditioning. In the Estes analysis, only cues produced by responses currently being executed acquire control over the reaction $\left(S_{1}\right)$, whereas in the present analysis $S^{I}$ as well as $S_{I}$ may acquire control over the reaction. Too, in the Estes analysis, once PRF animals begin to respond rapidly on all trials, i.e., no longer make competing responses in the alley, then the same response-produced cues occur for PRF and CRF animals. In the present analysis, the possibility was entertained that goalbox behaviors might, independently of alley behaviors, give 
rise to distinctive response-produced cues.

There was no indication in the present results that reward-produced cues influenced behavior in extinction following PRF training; however, this influence may have gone undetected here. The present results suggest that $S^{I}$ controlled behavior in extinction about as much as $\mathrm{S}^{\mathrm{N}}$ following PRF training, at least at ITIs up to $30 \mathrm{~min}$. Too, $\mathrm{S}^{\mathrm{N}}$ was found to control behavior in extinction following PRF training at an ITI as long as $1 \mathrm{~h}$. The present results do not indicate whether or not $S^{I}$ and $S^{N}$ are active at a 24-h ITI. Previous data, however (e.g., Capaldi \& Capaldi, 1970; Capaldi \& Morris, in press), suggest $S N$ to be active at a 24 h ITI.

Stimulus traces have been assumed to decay to zero within a few seconds (e.g., Hull, 1952) or a few minutes (e.g., Rothkopf, 1955). In some instances (e.g., Amsel, 1967; Gonzalez \& Bitterman, 1969), investigations employing a particular ITI have been reported, and it has been suggested that traces decay to zero within the ITI employed. Even in these cases, it is clear that traces are not considered to remain active for as long as $1 \mathrm{~h}$ or perhaps even $30 \mathrm{~min}$. Embodied in the very idea of traces, then, is the notion of rapid decay. However, the present data indicate that $S^{I}$ and particularly $S^{N}$ do not decay rapidly-indeed, on the basis of the present data, it cannot be asserted that these stimuli do in fact decay to zero beyond a $30-\mathrm{min}$ and a $1-\mathrm{h}$ ITI, respectively. Inasmuch as definite evidence was provided here that $\mathrm{S}^{\mathrm{I}}$ and $S^{N}$ are active at ITIs of $30 \mathrm{~min}$ and $1 \mathrm{~h}$, respectively, it seems permissible, if not indicated, to describe these stimuli as memories. However, this view does not preclude the possibility that goal events and response events may also give rise to fading traces.

\section{REFERENCES}

Amsel, A. The role of frustrative nonreward in noncontinuous reward situations. Psychological Bulletin, 1958, 55, 102-119.

Amsel, A. Partial reinforcement effect on vigor and persístence: Advances in frustration theory derived from a variety of within-subjects experiments. In K. W. Spence and J. T. Spence (Eds.), The psychology of learning and motivation: Advances in research and theory. Vol. 1. New York: Academic Press, 1967.

Amsel, A., Wong, P. T. P., \& Traupmann, K. L. Short-term and long-term factors in extinction and durable persistence. Journal of Experimental Psychology, 1971, 90, 90-95.

Capaldi, E. J. A sequential hypothesis of instrumental learning. In K. W. Spence and J. T. Spence (Eds.), The psychology of learning and motivation: Advances in research and theory. Vol. 1. New York: Academic Press, 1967.

Capaldi, E. J. Partial reinforcement: An hypothesis of sequential effects. Psychological R eview, 1966, 73, 459-477.

Capaldi, E, J., Berg, R. F.. \& Spariing, D. L. Trial spacing and emotionality in the rat. Journal of Comparative \& Physiological Psychology, 1971, 76, 290-299.

Capaldi, E. J., \& Hart, D. Influence of a small number of partial reinforcement training trials on resistance to extinction. Journal of Experimental Psychology, 1962, 64, 166-172.

Capaldi, E. J., \& Morris, M. D. Reward schedule effects in extinction: Intertrial interval, memory and memory retrieval Learning \& Motivation, in press.

Capaldi, E. J., Turnex, L., \& Wynn, W. H. Decremental and facilitative effects in the straight-alley runway under partial reinforcement. Journal of Comparative \& Physiological Psychology, 1962, 55, 545-549.

Estes, W. K. The statistical approach to learning theory. In $S$. Koch (Ed.), Psychology: $A$ study of a science. Vol. 2. New York: McGraw-Hill, 1959.

Gonzalez, R. C., \& Bitterman, M. E. Spaced-trials partial reinforcement effects as a function of contrast. Journal of Comparative \& Physiological Psychology, 1969, 67, 94-103.

Hull, C. L. A behavior system. New Haven: Yale University

Press, 1952

Rothkopf, E. Z. Distribution of practice and the temporal decay of response-produced stimuli. Joumal of Experimental Psychology, 1955, 49, 32-38

Teichner, W. H. Experimental extinction as a function of the intertrial interval during conditioning and extinction. Journal of Experimental Psychology, 1952, 44, 170-178.

Tyler, D. W., Wortz, E. C., \& Bitterman, M. E. The effect of random and alternating partial reinforcement on resistance to extinction in the rat. American Journal of Psychology, 1953, $66,57-65$.

(Received for publication March 25, 1974; accepted June 28,1974 .) 